\title{
Deep Learning Methods to Predict Mortality in COVID-19 Patients: A Rapid Scoping Review
}

\author{
Mahanazuddin SYED ${ }^{\mathrm{a}, 1}$, Shorabuddin SYED ${ }^{\mathrm{a}}$, Kevin SEXTON ${ }^{\mathrm{a}}$, Melody L. GREER ${ }^{\mathrm{a}}$, \\ Meredith ZOZUS ${ }^{\mathrm{b}}$, Sudeepa BHATTACHARYYAc ${ }^{\mathrm{c}}$, Farhanuddin SYED ${ }^{\mathrm{d}}$ and Fred \\ PRIOR $^{\mathrm{a}}$ \\ ${ }^{a}$ Department of Biomedical Informatics, University of Arkansas for Medical Sciences, \\ Little Rock, AR, USA. \\ ${ }^{\mathrm{b}}$ Department of Population Health Sciences, University of Texas Health Science \\ Center at San Antonio, San Antonio, TX, USA. \\ ${ }^{\mathrm{c}}$ Department of Biological Sciences and Arkansas Biosciences Institute, Arkansas State \\ University, Jonesboro, AR, USA. \\ ${ }^{\mathrm{d}}$ College of Medicine, Shadan Institute of Medical Sciences, Hyderabad, TS, IN.
}

\begin{abstract}
The ongoing COVID-19 pandemic has become the most impactful pandemic of the past century. The SARS-CoV-2 virus has spread rapidly across the globe affecting and straining global health systems. More than 2 million people have died from COVID-19 (as of 30 January 2021). To lessen the pandemic's impact, advanced methods such as Artificial Intelligence models are proposed to predict mortality, morbidity, disease severity, and other outcomes and sequelae. We performed a rapid scoping literature review to identify the deep learning techniques that have been applied to predict hospital mortality in COVID-19 patients. Our review findings provide insights on the important deep learning models, data types, and features that have been reported in the literature. These summary findings will help scientists build reliable and accurate models for better intervention strategies for predicting mortality in current and future pandemic situations.
\end{abstract}

Keywords. COVID-19, Deep Learning, Pandemic, Mortality, Death, Scoping Review.

\section{Introduction}

The novel coronavirus (COVID-19) disease has swiftly become a significant threat to global health [1]. As of January 30 2021, there have been more than 102 million cases confirmed worldwide and greater than 2.2 million fatalities due to COVID-19 [2]. The US is now the epicenter of the outbreak with more than 26 million cases and over 438,000 deaths due to the virus [2]. The pandemic's rapid spread has forced healthcare systems to allocate the resources thoughtfully and early detect the high-risk patients, thereby improving the patient outcomes [3].

\footnotetext{
${ }^{1}$ Corresponding Author, Mahanazuddin Syed, Department of Biomedical Informatics, University of Arkansas for Medical Sciences, Little Rock, AR, USA; E-mail: msyed2@uams.edu.
} 
Artificial Intelligence (AI) prediction models allow risk stratification of the patients based on clinical variables collected during hospital admission to triage the vulnerable population [4] effectively. To date, several studies have reported prediction models based on Machine Learning (ML) and Deep Learning (DL) that could predict COVID-19 disease severity and mortality [4-6]. These models heavily rely on the heterogeneous data types including imaging and unstructured notes collected during the course of hospitalizations. DL techniques has proven to have better accuracy when compared to traditional ML models and have helped to answer many questions related to virus evolution, predictor variables, and outcomes [7]. Few published studies have reviewed the literature that applied AI techniques to address the pandemic, covering multiple areas of epidemiology, diagnostics, and disease progression [1;3]. However, in this study, given the current pandemic situation and urgent need to quickly disseminate knowledge, we aimed to provide a rapid scoping review on the reported characteristics from the literature that used DL methods to predict hospital mortality in COVID-19 patients.

\section{Methods}

This review followed the Preferred Reporting Items for Systematic Reviews and MetaAnalyses (PRISMA) framework extension for scoping reviews [8].

\subsection{Eligibility Criteria, Data Sources and Search Strategy}

This study focused on peer-reviewed publications that applied DL algorithms to predict hospital mortality in COVID-19 patients. The PubMed database was searched for articles published in English, peer-reviewed, and/or conference proceedings available from Dec 1, 2019, through Dec 31, 2020. The search syntax was built with a broad set of keywords related to "COVID-19", "mortality", and "artificial intelligence" from the literature [3; 9]. Figure 1 illustrates the process of identifying eligible publications.

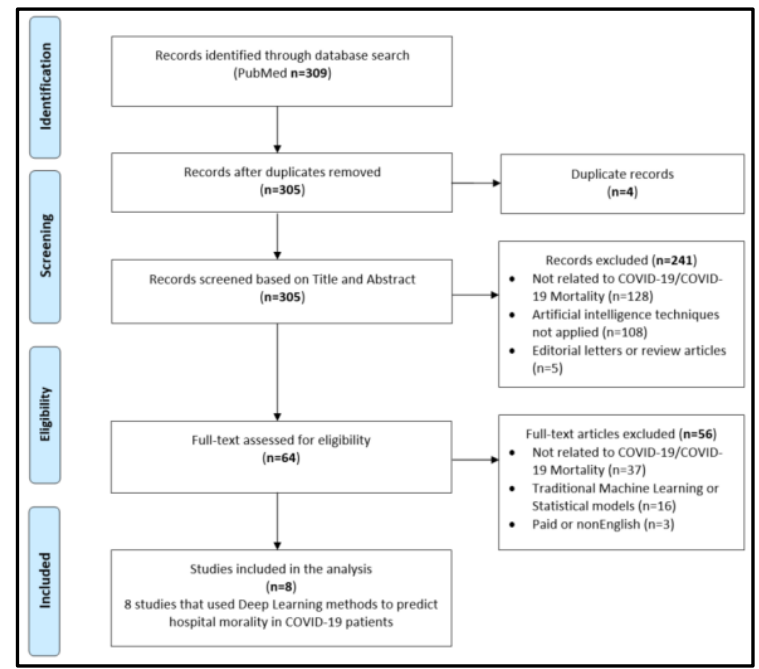

Figure 1. PRISMA (Preferred Reporting Items for Systematic Reviews and Meta-Analysis) flow diagram of systematic identification, screening, eligibility, and inclusion of publications that used Deep Learning techniques to predict hospital mortality in COVID-19 patients. 
Following the systematic search process, 309 publications were retrieved. Of that, 4 duplicate publications were removed, leaving 305 relevant articles for title and abstract screening. Two reviewers (MS and SS) screened the articles independently, following which 241 articles were removed, and 64 publications were retained for a full-text assessment. These studies were further assessed for eligibility, resulting in 8 articles that were included in the final analysis. Disagreements between reviewers were resolved by an independent review by a third reviewer (FS).

\section{Results}

Eight articles met the inclusion criteria, and were selected for further analyses. Of these, one article used a federated Multilayer Perceptron (MLP) model, one study used a combination of both traditional ML and DL methods. Two articles have augmented the model by adding radiology features along with the clinical features. Most of the studies have used comorbidities as a feature in the model. Three articles reported Area Under the Receiver Operating Characteristics (AUROC) that is greater than 90\%. All 8 studies used demographic variables in the model. Table 1 has details of individual studies.

Table 1. Details of 8 studies that used Deep Learning (DL) methods to predict hospital mortality in COVID19 patients.

\begin{tabular}{|c|c|c|c|}
\hline Study/Aim & Variables Used & Data Size \& Dates & Model Performance \\
\hline $\begin{array}{l}\text { Vaid et al [6]; To } \\
\text { predict mortality in } \\
\text { COVID-19 patients } \\
\text { within seven days of } \\
\text { hospital admission. }\end{array}$ & $\begin{array}{l}\text { Age, gender, ethnicity, } \\
\text { race, and past medical } \\
\text { history. }\end{array}$ & $\begin{array}{l}4029 \text { patients from } 5 \\
\text { hospitals; March } 15 \\
2020 \text { to May } 222020\end{array}$ & $\begin{array}{l}\text { Federated MLP best } \\
\text { performer with AUC } \\
\text { over } 90 \% \text {. }\end{array}$ \\
\hline $\begin{array}{l}\text { Ko et al [10]; To } \\
\text { predict in-hospital } \\
\text { mortality using routine } \\
\text { blood sample. }\end{array}$ & $\begin{array}{l}\text { Age, gender, and } 28 \\
\text { blood biomarkers. }\end{array}$ & $\begin{array}{l}467 \text { COVID-19 } \\
\text { patients; January } 2020 \\
\text { to July } 2020\end{array}$ & $\begin{array}{l}\text { EDRnet Accuracy of } \\
92 \%\end{array}$ \\
\hline $\begin{array}{l}\text { Abdulaal et al [11]; To } \\
\text { develop model that } \\
\text { predicts death during } \\
\text { admission with } \\
\text { COVID-19. }\end{array}$ & $\begin{array}{l}\text { Demographics, } \\
\text { comorbidities, } \\
\text { symptomatology, and } \\
\text { days of symptoms. }\end{array}$ & $\begin{array}{l}398 \text { patients; March } 1 \\
2020 \text { to April } 242020\end{array}$ & ANN AUROC 92.6\% \\
\hline $\begin{array}{l}\text { Ning et al [12]; To } \\
\text { predict COVID-19 } \\
\text { morbidity and } \\
\text { mortality. }\end{array}$ & $\begin{array}{l}\text { Demographics, Clinical } \\
\text { features, and CT } \\
\text { images. }\end{array}$ & 1521 patients; & HUST-19 AUC 0.856 \\
\hline $\begin{array}{l}\text { Kimura-Sandoval et al } \\
{[13] \text {; To predict }} \\
\text { mortality and } \\
\text { mechanical ventilation. }\end{array}$ & $\begin{array}{l}\text { Demographics, clinical, } \\
\text { biochemical, and } \\
\text { radiological data. }\end{array}$ & $\begin{array}{l}166 \text { patients; April } 1 \\
2020 \text { to April } 202020\end{array}$ & $\begin{array}{l}\text { AI model AUC of } 0.88 \\
\text { (best reported) }\end{array}$ \\
\hline
\end{tabular}




\begin{tabular}{llll}
\hline $\begin{array}{l}\text { Li et al [14]; To } \\
\text { predict ICU admission } \\
\text { and in-hospital } \\
\text { mortality }\end{array}$ & $\begin{array}{l}\text { Demographics, chronic } \\
\text { comorbidities, vital } \\
\text { signs, symptoms, and } \\
\text { lab tests. }\end{array}$ & $\begin{array}{l}\text { 5,766 patients under } \\
\text { investigation for } \\
\text { COVID-19; February } \\
7 \text { 2020 to May 4 2020 }\end{array}$ & $\begin{array}{l}\text { DL model with AUC of } \\
0.844\end{array}$ \\
\hline $\begin{array}{l}\text { Zhu et al [15]; To } \\
\text { predict the likelihood } \\
\text { of mortality and } \\
\text { identify top predictor } \\
\text { variables. }\end{array}$ & $\begin{array}{l}\text { Demographics, } \\
\text { comorbidities, vital } \\
\text { signs, symptoms, and } \\
\text { laboratory tests. }\end{array}$ & $\begin{array}{l}\text { 181 patients with } \\
\text { confirmed COVID-19 }\end{array}$ & $\begin{array}{l}\text { NN model with AUC of } \\
0.968 \text { with top 5 clinical } \\
\text { variables. }\end{array}$ \\
\hline $\begin{array}{l}\text { Abdulaal et al [16]; To } \\
\text { create a point-of- } \\
\text { admission mortality } \\
\text { risk scoring system. }\end{array}$ & $\begin{array}{l}\text { Demographics, } \\
\text { comorbidities, smoking } \\
\text { history, and presenting }\end{array}$ & 2020 to April 24 2020 & $\begin{array}{l}\text { ANN accuracy in } \\
\text { predicting patient } \\
\text { specific mortality was } \\
86.25 \%\end{array}$ \\
\hline
\end{tabular}

\section{Discussion and Recommendations}

DL subset of AI have become an important technology in health-related crisis management such as COVID-19 pandemic [17]. DL methods can be effectively used to predict hospital mortality among COVID-19 patients. In general, DL algorithms require large volumes of data for training, which helps to increase the model performance. In our review, most of the studies used smaller data sets and very few studies had larger data sets. To overcome data size issues, Vaid et al [6] proposed a federated learning framework model trained at local sites, and then model parameters are exchanged without sharing sensitive patient data. The proposed MLP federated model reported to have an Area under the Curve (AUC) greater than 90\%. This suggests newer studies should avoid data size limitations by collaborating in consortia and utilizing federated architecture models. The large data sets being collected by national and international collaborations such as National COVID Cohort Collaborative (N3C) will have sufficient data to train and develop robust predictive models [18]. In the study by Ko et al [10] the authors developed an ensemble model by combining DL and traditional ML to improve the performance. The model EDRnet achieved an accuracy of 92\% using 28 blood biomarkers, age, and gender as predictor variables.

Ning et al [12] and Kimura-Sandoval et al [13] proposed HUST-19 and AI models respectively that takes advantage of both imaging and clinical data. The model accuracy reported in both the studies was promising. In our review, most models performed well, one study by Zhu et al [15] reported the best AUC of 0.968 for predicting mortality using only 5 clinical variables (D-dimer, oxygen index, neutrophil to lymphocyte ratio, Creactive protein, and lactate dehydrogenase). The author further claims these variables are associated with mortality based on feature importance ranking. We recommend that future studies should try to identify a complete list of effective variables and their association with the mortality for better prediction.

There are some inherent limitations of the present rapid scoping review. First, there is the possibility of studies missed due to the search methodology. Second, lack of critical analysis of the risk of bias may weaken the attempts at understanding the data. However, the urgent need to understand the models, variables, and characteristics obtained from results provide reasonable evidence on the process analyzed. 


\section{Conclusion}

In this review, we summarized the literature that utilized DL methods to predict hospital mortality in COVID-19 patients. Our study provides insights into the DL techniques to use decision support tools in predicting hospital mortality. In addition, highlighting important variables and models that aid in clinical and translational research.

\section{Acknowledgment}

This study was supported in part by the Translational Research Institute (TRI), grant UL1 TR003107 received from the National Center for Advancing Translational Sciences of the National Institutes of Health (NIH). The study content is solely the responsibility of the authors and does not necessarily represent the official views of the NIH.

\section{References}

[1] Wynants L, et al. Prediction models for diagnosis and prognosis of covid-19: systematic review and critical appraisal, BMJ 2020;369:m1328.

[2] Dong E, Du H and Gardner L. An interactive web-based dashboard to track COVID-19 in real time, Lancet Infect Dis 2020;20:533-534.

[3] Syeda HB et al. Role of Machine Learning Techniques to Tackle the COVID-19 Crisis: Systematic Review, JMIR Med Inform 2021;9:e23811.

[4] Wollenstein-Betech S, Cassandras GC and Paschalidis IC. Personalized predictive models for symptomatic COVID-19 patients using basic preconditions: Hospitalizations, mortality, and the need for an ICU or ventilator, Int J Med Inform 2020;142:104258.

[5] Yadaw AS et al. Clinical features of COVID-19 mortality: development and validation of a clinical prediction model, Lancet Digit Health 2020;2:e516-e525.

[6] Vaid A et al. Federated Learning of Electronic Health Records Improves Mortality Prediction in Patients Hospitalized with COVID-19, JMIR Med Inform 2020.

[7] Cao C et al. Deep Learning and Its Applications in Biomedicine, Genomics, proteomics \& bioinformatics 2018; $16: 17-32$.

[8] PRISMA Extension for Scoping Reviews (PRISMA-ScR): Checklist and Explanation, Annals of Internal Medicine 2018;169:467-473.

[9] Yang L et al. Risk factors for predicting mortality of COVID-19 patients: A systematic review and metaanalysis, PLoS One 2020;15:e0243124-e0243124.

[10] Ko H et al. An Artificial Intelligence Model to Predict the Mortality of COVID-19 Patients at Hospital Admission Time Using Routine Blood Samples: Development and Validation of an Ensemble Model, J Med Internet Res 2020;22:e25442.

[11] Abdulaal A et al. Comparison of deep learning with regression analysis in creating predictive models for SARS-CoV-2 outcomes, BMC Med Inform Decis Mak 2020;20:299.

[12] Ning W et al. Open resource of clinical data from patients with pneumonia for the prediction of COVID19 outcomes via deep learning, Nat Biomed Eng 2020;4:1197-1207.

[13] Kimura-Sandoval $\mathrm{Y}$ et al. Validation of Chest Computed Tomography Artificial Intelligence to Determine the Requirement for Mechanical Ventilation and Risk of Mortality in Hospitalized Coronavirus Disease-19 Patients in a Tertiary Care Center In Mexico City, Rev Invest Clin 2020.

[14] Li X et al. Deep learning prediction of likelihood of ICU admission and mortality in COVID-19 patients using clinical variables, PeerJ 2020;8:e10337.

[15] Zhu JS et al. Deep-learning artificial intelligence analysis of clinical variables predicts mortality in COVID-19 patients, J Am Coll Emerg Physicians Open 2020;1:1364-1373.

[16] Abdulaal A et al. Prognostic Modeling of COVID-19 Using Artificial Intelligence in the United Kingdom: Model Development and Validation, J Med Internet Res 2020;22:e20259.

[17] Benis A, Tamburis O, Chronaki C and Moen A. One Digital Health: A Unified Framework for Future Health Ecosystems, J Med Internet Res 2021;23:e22189.

[18] Melissa H, Christopher C and G. Kenneth. The National COVID Cohort Collaborative (N3C): Rationale, Design, Infrastructure, and Deployment, J Am Med Inform Assoc 2020:ocaa196. 